\title{
Estado actual del clareamiento dental
}

\section{Current state of dental bleaching}

\begin{abstract}
Resumen:
Uno de los tratamientos más popularizados y exigidos por los pacientes es el de clareamiento dental; este procedimiento es de resultados bastante previsibles, fácil de realizar y no invasivo, pero es uno de los procedimientos que más dudas tiene el profesional al realizarlo, muchas veces nos valemos de técnicas y procedimientos que carecen de sustentos científicos y conllevan a resultados poco satisfactorios. En este artículo se realiza una revisión de la literatura y se formula una secuencia que puede orientar al profesional para realizar un adecuado protocolo en el clareamiento dental en diferentes situaciones clínicas. Es fundamental no sólo cuando se realiza procedimientos de clareamiento dental sino en cualquier procedimiento un diagnóstico correcto, así como poder ofrecer un enfoque desde varias perspectivas interrelacionando las diferentes especialidades de la odontología con la finalidad de obtener los mejores resultados posibles.

Palabras Clave: Peróxido de hidrógeno; Oxidación; Terapias de blanqueamiento dental; Luz de activación.
\end{abstract}

\section{Abstract:}

One of the most popularized treatments required by patients is the dental bleaching; this procedure has fairly predictable results, easy to perform, noninvasive, but is one of the procedures that the profesional has more doubts to do it, sometimes we make use of techniques and procedures that lack scientific support and lead to unsatisfactory results. This article presents a literature review and is performed a sequence that can guide the professional for proper protocol in the dental bleaching in different clinical situations. It is essential to make a correct diagnosis, not only when dental whitening procedures but in any proceedings, as well as to offer an approach from various perspectives interrelating the various specialties of dentistry in order to achieve the best possible results.

Keywords: Hydrogen peroxide; Oxidation; Teeth whitening therapies; Activation light.

\section{Introducción}

Dentro de la odontología actual los procedimientos estéticos son cada vez más frecuentes obedeciendo a cánones de belleza impuestos, por consiguiente el profesional debe de estar capacitado para realizar correctamente este tipo de procedimientos; dentro de estos procedimientos se encuentra incluido los procedimientos de clareamiento dental ${ }^{1}$. Los procedimientos de clareamiento dental se han popularizado bastante en estos últimos años sin embargo muchos profesionales tienen dudas de cómo aplicar estas técnicas por desconocimiento de los procedimientos adecuados; ya sea realizando un clareamiento dental utilizando la técnica ambulatoria o en el consultorio. Surgen dudas en la cantidad de aplicaciones del gel, por cuanto tiempo se debe aplicar el gel, que concentración se debe utilizar, que hacer en caso de sensibilidad ${ }^{2}$.

Existen varios materiales que se utilizan actualmente para realizar el procedimiento clareamiento dental como son el peróxido de hidrógeno, el peróxido de carbamida y el perborato de sodio, muchas veces mezclados con sustancias remineralizantes. De todos estos materiales es el oxígeno liberado como principio activo quien realiza la función de clareamiento iniciando el proceso de degradación de moléculas orgánicas complejas causantes de las alteraciones de color. Es a través de este proceso de oxidación que se logra moléculas de carbono hidrófilas no pigmentadas siendo este producto ideal; si se persiste en la exposición del esmalte al peróxido se generaría una descomposición de la matriz orgánica produciéndose una pérdida de proteínas del esmalte ${ }^{3-5}$.

La diferencia entre el peróxido de hidrógeno y el peróxido de carbamida radica en que el primero sufre su proceso de oxidación es decir su efecto clareador en un periodo de tiempo entre 15 a 60 minutos y el peróxido de carbamida libera el $50 \%$ de su principio activo en las primeras dos horas, y el otro 50\% de una manera sostenida hasta en las 10 horas subsecuentes aproximadamen$\mathrm{te}^{6}$. Los radicales libres generados en el proceso de clareamiento son capaces de reaccionar con proteínas, lípidos y áci-
César Lamas-Lara ${ }^{1}$, Sergio AlvaradoMenacho², Liliana Terán-Casafranca ${ }^{2}$, Giselle Angulo de la Vega ${ }^{3}$, Angie Hidalgo-Castañeda ${ }^{4}$

1. Facultad de Odontología de la Universidad San Martin de Porres, filial Norte, Perú.

2. Departamento Académico Estomatología Rehabilitadora de la Facultad de Odontología de la Universidad Nacional Mayor de San Marcos, Perú.

3. Segunda especialidad de Rehabilitación Oral de la Unidad de Postgrado de la Facultad de Odontología de la Universidad Nacional Mayor de San Marcos, Perú.

4. Escuela de Pregrado de Odontología de la Universidad Católica Los Ángeles Chimbote, Perú. Correspondencia:

CD. César Lamas Lara

Santa Honorata 415. Urb. Pando, 3era Etapa. San Miguel. Lima 1. Perú

Correo electrónico: cesar2579@hotmail.com

\section{Coautores}

Alvarado-Menacho: salvarado4@hotmail.com Terán-Casafranca: teranliliana@hotmail.com

Fecha de recepción: 11-08-14

Fecha de aceptación: 31-10-14

dos nucléicos causando daño celular. Estudios indican que la genotoxicidad y carcinogenicidad sólo se producen en concentraciones altas que no se logran durante el clareamiento dental ${ }^{7,8}$.

Otros efectos de los geles clareadores se han descrito en la literatura como sensibilidad dental e irritación gingival, alteraciones de la superficie del esmalte y efectos sobre materiales restauradores. La mayoría de estos efectos locales son dependientes de la concentración del producto a ser aplicado?

Los compuestos orgánicos que se fijan a las estructuras dentarias (manchas) tienen características que hacen reflejar longitudes de onda que al ojo humano son los responsables de la tinción de las piezas dentarias. Se supone que los geles de clareamiento actúan destruyendo uno o más dobles enlaces de la cadena de carbono de la mancha o a través de la oxidación de otros entes químicos presentes en ella, obteniendo estructuras de carbono más cortas y con enlaces de carbono saturados, con la capacidad de absorber menos cantidad de 
luz, siendo estas menos perceptibles por el ojo humano ${ }^{10,11}$.

Dentro de las alteraciones de color que los dientes pueden presentar tenemos las causadas por pigmentaciones intrínsecas y pigmentaciones extrínsecas. Las pigmentaciones deben de ser analizadas según su permanencia ya que esto nos ayudará a elegir el tipo de agente clareador a utilizar, su concentración y duración del tratamiento. Las pigmentaciones pueden ser recientes, temporales o permanentes; si más tiempo tiene la pigmentación más difícil será eliminarla ${ }^{12}$.

Las pigmentaciones extrísencas se ven asociadas a la dieta (taninos alimenticios) o a hábitos (fumar, masticar tabaco, hoja de coca etc.). Las pigmentaciones intrínsecas pueden ser congénitas o adquiridas; las congénitas pueden asociarse a amelogénesis imperfecta, dentinogénesis imperfecta y las adquiridas en la fase preeruptiva podemos encontrar a las ocasionadas por fluorosis, tetraciclinas, etc. y en una fase posteruptiva puede ser ocasionada por un traumatismo dentario, hemorragia intrapulpar o necrosis ${ }^{13}$.

Las alteraciones de color por tetraciclina están clasificadas dentro de las causas intrínsecas pre eruptivas; el periodo de riesgo de esta alteración abarca todo aquello en donde se produzca la formación de tejido dentario. Entonces el período de riesgo comienza desde el segundo trimestre de desarrollo intrauterino hasta los 8 ańos de edad. Es suficiente el consumo de tetraciclinas por 3 días para ocasionar alteraciones de grado moderado a severo. Las tetraciclinas tienen una fijación por los iones de calcio, y esta unión entre las tetraciclinas y el calcio se incorpora a los dientes y al hueso. La exposición a la luz hace que se produzca una reacción cromógena, por este motivo se aprecia este tipo de tinciones con más intensidad en la cara vestibular de las piezas dentarias ${ }^{14,15}$.

Una de las propiedades de las tetraciclinas es la fluorescencia, cuando los dientes son expuestos a una luz ultravioleta estos se tornan excesivamente brillantes, este es el medio de diagnóstico clínico de esta alteración.

El objetivo de este artículo es realizar una revisión de las diferentes técnicas del clareamiento dental así como establecer protocolos básicos en cada caso basándonos en la información científica con la que contamos actualmente.

\section{Técnicas de clareamiento dental}

En la actualidad existen diferentes métodos o técnicas para realizar el clareamiento dental pero podemos agruparlas en Clareamiento Externo cuando aplicamos el gel clareador sobre las superficies externas de las piezas dentarias y Clareamiento Interno cuando el agente clareador es aplicado en la cámara pulpar de dientes desvitalizados; también podemos mezclar ambas técnicas dependiendo de las circunstancias, como se ilustra en el cuadro $\mathrm{N}^{\circ} 1$. Dentro del clareamiento externo existe la técnica ambulatoria, en la cual el paciente cobra una vital importancia para la aplicación del gel en su hogar siempre bajo la supervisión del profesional; y la técnica de consultorio que es donde el profesional es el que aplica el gel clareador y tiene el total control sobre los materiales empleados.

Dentro del clareamiento interno tenemos la técnica inmediata en donde se utilizan geles de alta concentración aplicado en la cámara pulpar y se eliminan en la misma sesión; y la técnica mediata en la cual se deja el gel dentro de la cámara durante un periodo de días determinado para que realice su efecto.

Cuadro 1. Técnicas de Clareamiento Dental

\begin{tabular}{ll}
\hline Clareamiento externo & Casero o ambulatório \\
& Consultorio \\
& Consultorio + Casero \\
Inmediata & Mediata (Walking Bleaching) \\
& Inmediata + Mediata \\
Clareamiento externo & \\
+ interno & \\
\hline
\end{tabular}

a) Clareamiento externo ambulatorio o domiciliario

El clareamiento ambulatorio debe estar siempre supervisado por el profesional que le proporciona e instruye al paciente en la aplicación del agente clareador. Es necesario utilizar una cubeta individualizada utilizando una lámina de acetato de $0.6 \mathrm{~mm}$ de espesor termoformada por una plastificadora. Para realizar estas cubetas se realizan impresiones simples al paciente para la obtención de modelos de yeso. Estos modelos deben de tener ciertas características como carecer de fondo de surco y en el caso del modelo superior carecer del paladar, ya que la presencia de estas estructuras puede dificultar la confección de las cubetas (Fig.1).

Una vez confeccionada la cubeta se recorta tratando de seguir el contorno de la encía evitando estructuras como los frenillos las cuales la cubeta podría lesionar (Fig. 2); se instruye al paciente como aplicar el gel, este gel debe ser aplicado en la superficie interna de la cubeta en lo que vendría ser la cara vestibular de las piezas dentarias; una pequeña cantidad en el centro de la cara vestibular, (Fig. 3) ya que si colocamos más cantidad esta será expulsada por fuera de la cubeta hacia la encía pudiendo ocasionar molestias al paciente.

El uso de reservorios creados sobre el modelo aplicando espaciadores para poder aplicar el gel de clareamiento no es necesario ya que al confeccionar la cubeta esta presenta una separación sobre el modelo de yeso, espacio suficiente para la cantidad del gel. El tiempo de contacto del gel sobre las superficies de los dientes se establece entre 6 a 8 horas en cada aplicación.

Hoy en día el clareamiento con cubetas resulta una opción efectiva para el tratamiento de la mayoría de alteraciones de color que pueden presentar las piezas dentarias, antes de realizar un tratamiento invasivo como son carillas o coronas cerámicas. Para el clareamiento con cubetas se han utilizado diferentes concentraciones de peróxido de carbamida considerando como seguras la aplicación en concentraciones de 10\% y $16 \%$ en un periodo de 3 semanas $^{16}$.

Generalmente este gel el paciente lo aplicará en las noches antes de dormir y estará con la cubeta hasta la mañana siguiente

Durante el tratamiento el paciente debe de realizar la higiene bucal acostumbrada y la limpieza de la cubeta para su próxima aplicación. También se puede utilizar peróxido de hidrógeno pero en bajas concentraciones $(3 \%, 4 \%, 6 \%$ y $7 \%)$. En concentraciones mayores $(35 \%$ de peróxido de carbamida), este gel puede ser empleado en el consultorio aplicado en la cubeta por un tiempo de $30 \mathrm{~min}$.

\section{b) Clareamiento externo de consul- torio}

Es muy común la técnica de blanqueamiento en el consultorio odontológico con fuente de activación por luz, sea estos LED, Arco de Plasma, Láser o Hibrida. No se sabe a ciencia cierta cuál es el efecto real de la luz sobre los geles de clareamiento, pero se puede deducir que las lámparas de clareamiento tienen alta potencia (alrededor de $1500 \mathrm{mw} / \mathrm{cm}^{2}$ o superiores) y generan un incremento en la temperatura de la superficie expuesta, por consiguiente generan un aumento de temperatura del gel de clareamiento, 
esto acelera la reacción de oxidación del peróxido; si esto es cierto la exposición a una fuente de luz reduciría el tiempo de exposición del gel en la superficie dental; pero no se ha demostrado que al utilizar estos equipos se logren mejores resultados que al realizar el clareamiento sin ellos. Se puede obtener similares resultados fotopolimerizando los geles de clareamiento así como dejándolos actuar sin la necesidad de la activación con luz ${ }^{17-22}$. Antes de la aplicación del gel clareador debemos de realizar una limpieza de las superficies a tratar por el profesional, posteriormente ya que el gel clareador es altamente caustico debemos de proteger las estructuras blandas realizando un aislamiento absoluto o un aislamiento relativo con separadores de carrillo y lengua, barreras gingivales, rollos de algodón y eyectores de saliva (Fig. 4).

Una vez colocadas las barreras de seguridad se procede a la aplicación del gel; generalmente se emplea peróxido de hidrógeno al 35\% - 37\%, este gel se aplica sobre las superficies vestibulares de los dientes en la parte central, no es necesario esparcirlo por toda la cara vestibular ya que estos geles tienen una gran capacidad de difusión sobre el esmalte y la dentina. El gel de clareamiento debe de estar en contacto con la superficie del esmalte por un tiempo de $30 \mathrm{~min}$ (Fig. 5). Una vez transcurrido en tiempo respectivo se retira el gel con un eyector, de preferencia con uno endodóntico, tratando de no humedecer las superficies; se puede repetir el procedimiento 3 veces en una cita, realizándose entre 2 a 3 citas en intervalos de 1 semana.

El clareamiento dental realizado correctamente no afecta la integridad de las piezas dentarias, no afecta la microdureza superficial del esmalte o de la dentina de manera significativa ni tampoco es un desencadenante para la aparición de lesiones de caries. Si bien es cierto hay una reducción de la microdureza superficial esta se ve expresada en caras no funcionales de las piezas dentarias que es generalmente en la superficies en donde se realiza el procedimiento de clareamiento (cara vestibular) y esta reducción no es significativa además la capacidad remineralizante de la saliva eliminaría esta reducción en corto tiempo ${ }^{23}$.

Es recomendable realizar los procedimientos de clareamiento dental arcada por arcada, ya que es la mejor manera que podamos controlar los cambios obtenidos y el paciente puede apreciarlos de mejor manera, (Fig. 6).
En algunos casos el utilizar altas concentraciones de peróxido de hidrógeno (35\% a 37\%) puede ocasionar sensibilidad que generalmente es leve y temporal pero en algunos casos puede ocasionar molestias considerables. El tratamiento varía desde el uso de analgésicos antinflamatorios, hasta la aplicación de pastas desensibilizantes a base de fluoruros que obliteran los túbulos dentinarios o a base de nitrato de potasio que disminuyen la capacidad de las fibras nerviosas de transmitir el dolor ${ }^{24-26}$.

\section{c) Clareamiento interno técnica in- mediata}

Las causas de las discromías en dientes tratados endodónticamente pueden ser muy variadas, restos pulpares que se quedan dentro de la cámara pulpar luego de realizar el tratamiento de conductos, algunos materiales como gutapercha también pueden ocasionar tinciones, así como la difusión de los componentes sanguíneos dentro de los túbulos dentinarios, se consideran como los principales factores de las discromías $^{27,28}$.

En un blanqueamiento interno es muy importante la colocación de sellador biomecánico (sellador biológico y sellador mecánico) a nivel radicular que llegue a la unión cemento esmalte para prevenir la reabsorción cervical externa que puede ser producida por la acción del peróxido a ese nivel ${ }^{29}$.

Antes de realizar el clareamiento dental debemos de desobturar el conducto radicular desde la unión cemento esmalte $3 \mathrm{~mm}$ hacia apical; en el milímetro en contacto con la gutapercha colocaremos hidróxido de calcio en pasta (sellador biológico) que servirá como neutralizante a cualquier molécula del gel clareador que quiere acercarse a esa zona; y en los 2 milímetros restantes se colocará ionómero de vidrio de autocurado (sellador mecánico), para evitar cualquier tipo de desadaptación y posible microfiltración del peróxido en el conducto ${ }^{30}$.

Una vez aplicado el ionómero de vidrio se tiene que esperar una cita posterior (entre 48 a 72 horas para que el ionómero complete su fraguado) para iniciar el procedimiento de clareamiento. Se utilizará los mismos geles utilizados para la técnica de clareamiento externo en el consultorio (peróxido de hidrógeno al 35\% 37\%), aplicados en intervalos de 30 minutos, 3 aplicaciones por cita, entre 2 a 3 citas en intervalos de 1 semana. Para optimizar los resultados podemos realizar el clareamiento interno y externo de la pieza dentaria en la misma sesión (Fig. 7). Para mejorar la penetración del gel de peróxido dentro del esmalte dentario podemos aplicar ácido ortofosfórico a 37\% sobre las superficies dependiendo de la severidad de la discromía, (Fig. 8).

\section{d) Clareamiento interno técnica me- diata}

En esta técnica de clareamiento interno al igual que en el anterior es muy importante la confección de un buen tapón biológico ya que se ha relacionado la no confección de este con procesos de reabsorción radicular a nivel cervical. En este caso se utiliza generalmente peróxido de carbamida al $37 \%$ aplicado sobre la cámara pulpar y dejado dentro de la misma por un período de 7 días, 1 aplicación cada cita entre 2 a 3 citas, dependiendo de la severidad de la discromía.

Para mantener el gel en la cámara se utiliza una torunda de algodón (Fig.9) y se sella la cavidad con ionómero de vidrio, una vez transcurrido los 7 días se retira el ionómero, la torunda de algodón; se limpia la cavidad y se repite el procedimiento.

Una vez terminado el procedimiento es aconsejable aplicar hidróxido de calcio mezclado con suero fisiológico preparado en forma de pasta en la cámara pulpar por un período de 2 semanas para neutralizar el efecto de peróxido residual $^{31}$.

El realizar un clareamiento dental externo e interno en un diente tratado endodónticamente a veces es insuficiente para obtener resultados satisfactorios. El complementar técnicas de clareamiento con restauraciones de resina compuesta nos puede ayudar a optimizar nuestros resultados ${ }^{32}$, (Fig. 10).

\section{Discusión}

La eficacia del clareamiento depende más del tipo de tinción y del agente causal que de la ubicación de la mancha en la superficie o en lo profundo del espesor dentinario ya que se ha demostrado la gran capacidad de penetración del peróxido de carbamida a lo largo de todo su espesor ${ }^{33}$.

El algunos casos el combinar diferentes técnicas de clareamiento dental puede producir mejores resultados en nuestros tratamientos y está en el profesional elegir las mejores técnicas para optimizar sus resultados clínicos ${ }^{34,35}$.

El uso de peróxido de hidrógeno sólo o en combinación con el perborato de sodio puede afectar la microdureza y elasticidad así como el aumento de la rugosidad superficial. Esto se debe a que 


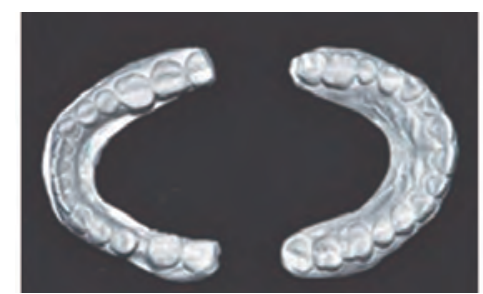

Fig. 1:

Modelos arcada superior e inferior para confección de cubetas de clareamiento.

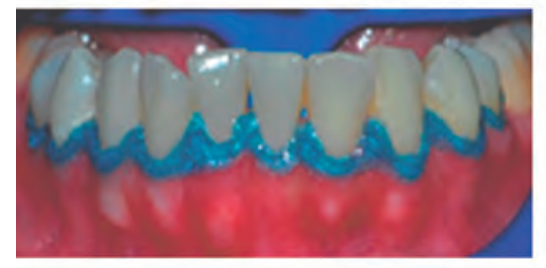

Fig. 4:

Colocación de la barrera gingival arcada inferior

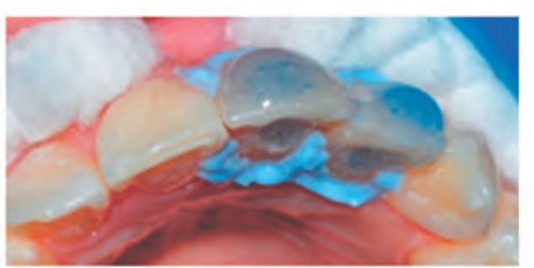

Fig. 7:

Realización del clareamiento externo e interno en simultáneo de las piezas 2.1 y 2.2

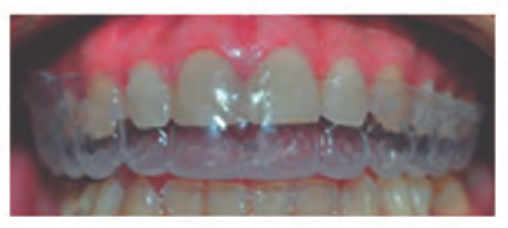

Fig. 2:

Prueba de la cubeta de clareamiento en boca.

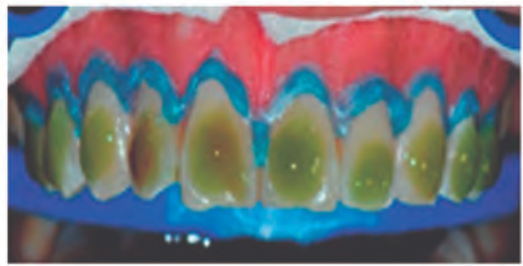

Fig. 5:

Aplicación del gel de peróxido de hidrogeno arcada superior

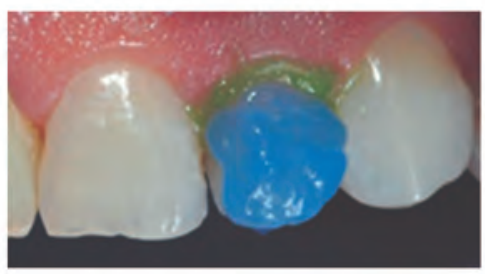

Fig. 8:

Aplicación de ácido ortofosfórico sobre las superficies a tratar.

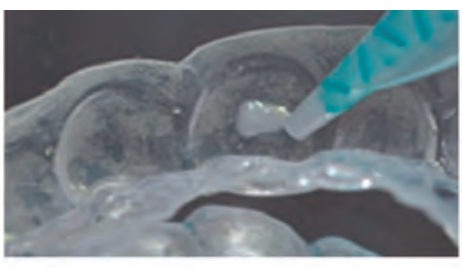

Fig. 3:

Aplicación del gel clareador en la cubeta

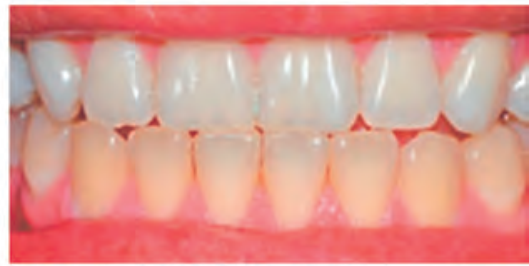

Fig. 6:

Vista de control comparación entre la arcada superior post clareamiento y arcada inferior antes del clareamiento.

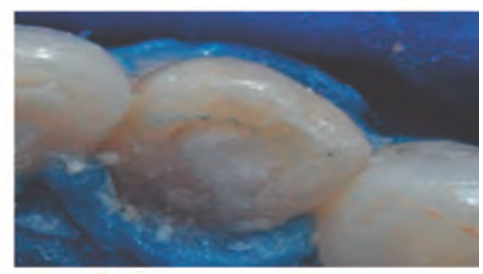

Fig. 9:

Aplicación de peróxido de carbamida dentro de la cámara pulpar con una torunda de algodón.

Tomado de La Revista Odontol. Sanmarquina; Alternativa de Restauración Estética en caso de discromía. 2010; 13(2): 39.

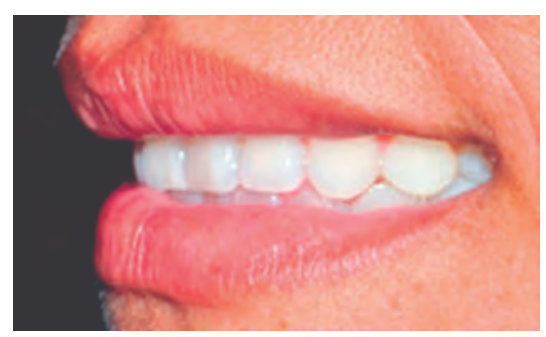

Fig. 10:

Vista final post clareamiento 
la aplicación del gel clareador sobre el esmalte puede promover la disolución de la matriz de este tejido altamente mineral; no obstante no se han registrado casos de fracturas dentarias asociadas al procedimiento de clareamiento dental ya que generalmente las áreas donde se aplican los geles de clareamiento son superficies no funcionales además de contar con la capacidad remineralizante de la saliva. Se menciona en la literatura que los geles de clareamiento a bajas concentraciones como el peróxido de carbamida al $10 \%$ tiene un mayor efecto negativo sobre la superficie que en profundidad y esto se puede deber a la presencia de la molécula de carbopol (espesante) que propicia una liberación lenta y sostenida de los radicales libres; posiblemente debido a esto sus efectos negativos en profundidad se vean disminuidos; a diferencia de los geles de altas concentraciones como son los geles de peróxido de hidrógeno al 35\% que por su rápida difusión y oxidación liberan gran cantidad de radicales libre ocasionando una disminución significativa en la microdureza del esmalte ${ }^{36-39}$.

Los agentes clareadores pueden reaccionar con la molécula orgánica que se encuentre disponible (agente inespecífico) por tal motivo también podrían reaccionar con las moléculas presentes en la restauración de resina aumentado la rugosidad superficial volviéndolas más susceptible a la adhesión de microorganismos. Sin embargo en la literatura es un tema controversial ya que existen investigaciones que mencionan resultado negativos en torno a la microdureza de las restauraciones de resina así como el aumento de la rugosidad superficial y otras que no reflejan disminución en sus valores ${ }^{40-42}$.

Para propiciar la apertura de los túbulos dentinarios y por consiguiente la mayor facilidad con la que el gel va a penetrar dentro de la dentina podemos utilizar hipoclorito de sodio al $1 \%$ asociado a ácido fosfórico al 37\%, o la aplicación de EDTA al $17 \%$ o solamente ácido ortofosfórico al $37 \%$, esto va a ocasionar la limpieza eficiente de la cámara pulpar así como la eliminación del barro dentinario $^{43,44}$.

Los geles de clareamiento pueden aumentar la solubilidad del ionómero de vidrio y otros cementos, además de reducir la fuerza de adhesión entre esmalte y materiales resinosos. Estudios actuales relacionan la acides de los geles de clareamiento a esta reducción, es decir entre más ácido sea el gel mayor generación de radicales libres que pueden afectar el proceso de adhesión por más tiempo. El tiempo de espera después del clareamiento para realizar restauraciones de resina compuesta varía de acuerdo a la literatura revisada pero se considera seguro esperar 2 semanas antes de realizar las restauraciones respectivas. Otro efecto indeseado es la reabsorción cervical externa y esto se da gracias a la gran capacidad de los radicales libre de difundirse por la dentina y llegar a la zona del ligamento periodontal en donde se inicia la reabsorción. Estudios demuestran que la asociación del clareamiento dental interno y un traumatismo previo aumenta el porcentaje de poder presentar este efecto indeseado. Se presume que la aplicación de los agentes clareadores asociado al calor, altas concentraciones o acides del agente pueden ser factores importantes en las reabsorciones radiculares ${ }^{8,45,46}$.

El principal efecto no deseado del clareaminento dental es la sensibilidad ya sea aplicando peróxido de hidrógeno o de carbamida; este efecto indeseado se puede producir porque el peróxido aumenta la permeabilidad del esmalte y la dentina y esto ocasiona el movimiento de los fluidos dentro de los túbulos dentinarios y por consiguiente sensibilidad post operatoria. La aplicación de desensibilizantes a base de nitrato de potasio al 5\% no interfiere con la eficacia clareadora del peróxido de hidrógeno al $35 \%$ ya que el efecto de este nitrato de potasio no actúa por obliteración tubular sino por repolarización de la fibras nerviosas; a diferencia de los desensibilizantes a base de fluoruros, pero a pesar de esto estudios reciente demuestran que la eficacia del clareamiento no se ve alterada por su aplicación ${ }^{23,24,47,48,49}$.

En relación a la aplicación del clareamiento en el consultorio con fuentes auxiliares de luz no se sabe realmente cuál es el efecto de la luz sobre los geles de clareamiento, se supone que estas lámparas generan un incremento en la temperatura del gel de clareamiento, esto acelera la reacción de oxidación del peróxido y por consiguiente la liberación de radicales libres; si esto es cierto la exposición a una fuente de luz reduciría el tiempo de exposición del gel en la superficie dental; pero no se ha demostrado que al utilizar estos equipos se logren mejores resultados que al realizar el clareamiento sin ellos ${ }^{12-17}$. En relación a las fuentes híbridas (LED - Láser), se dice que presentan mejores resultados al acelerar la reacción de oxidación generando menos calor (LED) y produciendo un efecto sedativo o terapéutico sobre la pulpa dental (láser). Sin embargo estudios demuestran que estos efectos ideales no serían muy diferentes que al aplicar otras fuentes de luz o incluso no aplicándolas. En relación a la cantidad de citas que se recomienda con la técnica de consultorio estudios demuestran que 1 sola sesión es insuficiente por lo que se recomienda la aplicación de 2 a 3 sesiones; esto dependiendo de la severidad y el tipo de tinción ${ }^{50,51}$.

\section{Conclusiones}

- El proceso de clareamiento ha ido evolucionando con el tiempo de diversas maneras, no sólo en lo concerniente a materiales sino en procedimientos; debemos de basarnos en evidencia científica sólida para realizar estos procedimientos.

- La fotoactivación de los geles de clareamiento es un tema controversial, hasta la actualidad no se sabe a ciencia cierta cuál es el efecto de las fuentes de luz sobre los geles de clareamiento pero está claro que podemos realizar procedimientos de clareamiento en el consultorio sin la necesidad de estas fuentes auxiliares obteniendo resultados satisfactorios.

- Es fundamental la buena confección de una tampón biológico cuando se realiza clareamiento interno sea la técnica mediata o inmediata, ya que la literatura demuestra un alto índice de reabsorción radicular por la no utilización del tapón o por su inadecuada confección.

- Es muy importante realizar un registro fotográfico adecuado antes, durante y después del clareamiento dental con la finalidad de poder ilustrar de una mejor manera al paciente los cambios obtenidos.

- Es fundamental un diagnóstico correcto no sólo cuando se realiza procedimientos de clareamiento dental, sino en cualquier procedimiento así como poder ofrecer un enfoque desde varias perspectivas interrelacionando las diferentes especialidades de la odontología con la finalidad de obtener los mejores resultados posibles.

\section{Referencias bibliográficas}

1. Lamas C, Alvarado S, Angulo G. Aclareamiento Dental en tinciones por tetraciclinas. Odontol Clín Cient. 2013;12(1):77-80.

2. Lamas C; Jara Y; Dominguez Y; Angulo G. Clareamiento Dental en el Consultorio. In Cres. 2012;3(1):131-8. 
3. Ben-Amar A, et al. Effect of mouthguard bleaching on enamel surface. Am J Dent. 1995;8(1):2932.

4. Bitter N, Sanders J. The effect of four bleaching agents on enamel surface. A sacanning electron microscopic study. Quintessence Int. 1993;24(11):817-24.

5. Posso S, Ramírez D, Rosas J, Güiza E. Comparación del blanqueamiento dental con peróxido de hidrógeno al $25 \%$ en consultorio, utilizando o no luz halógena (Zoom). Univ Odontol. 2010;29(62):19-25.

6. Haywood VB. Frequently asked questions about bleaching. Compend Contin Educ Dent. 2003;24(4A):324-38.

7. Suemori T, Kato J, Nakazawa T, Akashi G, Hirai Y. A new nonvital tooth bleaching method using titanium dioxide and 3.5\% hydrogen peroxide with a 405-nm diode laser or a halogen lamp. Laser Physics Letters. 2008;5(6):454-9.

8. Goldberg M, Grootveld M \& Lynch E. Undesirable and adverse effects of tooth-whitening products: a review. Clinical Oral Investigations. 2010;14(1):1-10.

9. Dahl JE, Pallesen U. Tooth bleaching a critical review of the biological aspects. Critical Reviews in Oral Biology and Medicine: An Official Publication of the American Association of Oral Biologists. 2003;14(4):292-304.

10. Joiner A. The bleaching of teeth: a review of the literature. J Dent. 2006;34(7);412-9.

11. Sulieman M, Addy M, MacDonald E, Rees JS. The effect of hydrogen peroxide concentration on the outcome of tooth whitening: an in vitro study. J Dent. 2004;32(4);295-9.

12. Goldstein R, Lancaster J. Survey of patient attitudes toward current esthetic procedures. J Prosthet Dent. 1984;52(6):775-80.

13. Grobler S, Kotze D. Relationship between enamel fluoride levels, degree of fluorosis and caries experience in communities with a nearly optimal and a high, fluoride level in drinking water. Caries Res 1986;20(3):284-8.

14. Cheek C, Haymenn H. Dental and oral discolorations associated with minocycline and other tetracycline analogs. J Esthet Dent 1999;11(1):43-7.

15. Nathoo S. The chemistry and mechanism of extrinsic and intrinsic discoloration. JADA 1997;128(suppl):6S-10S.

16. Meireles S, Heckmann S, Leida F, Santos I, Della Bona A, Demarco F. Efficacy and safety of $10 \%$ and $16 \%$ carbamide peroxide tooth-whitening gels: a randomized clinical trial. Oper Dent 2008;33(6):606-8. doi: 10.2341/07-150.

17. Gomes R, de Souza F, Lacerda C, Bambrilla C, Pascotto R. Avaliação clínica da eficiência do uso do sistema LED-laser, LED e luz halógena na ativaçáo do agente clareador em dentes vitalizados. Dental Press Estét 2008;5(2):6277

18. Carrasco L, Guerisoli D, Rocha M, Pécora J, Fröner I. Efficacy of intracoronal bleaching techniques with different light activation sources Int Endod J 2007; 40(3): 204-8

19. Marson F, Sensi L, Vieira L, Araújo E. Avaliação clínica do clareamento dental pela técnica no consultório. R Dental Press Estét 2007;4(4):50-60.

20. Alves E, Alves F, Campos E, Mathias P. Susceptibility to caries like lesions after dental bleaching with different techniques. Quintessence Int 2007;38(7):4049.

21. Ribeiro A, et al. Evaluation of tooth color after bleachingwith and without light-activation. Rev Odonto Cienc. 2011;26(3):247252.

22. Buchalla W, Attin T. External bleaching therapy with activation by heat, light or laser-A systematic review. Dent Mater. 2007;23:58696.

23. Rodrigues J, Erhardt MCG, Marchi GM, Pimenta LAF, Ambrosano GMB. Association effect of in-office and nightguard vital bleaching on dental enamel microhardness. Braz J Oral Sci. 2003;2(7):365-9.

24. Armênio RV, Fitarelli F, Armênio MF, Demarco FF, Reis A, Loguercio $\mathrm{AD}$. The effect of fluoride gel use on bleaching sensitivity: a double- blind randomized controlled clinical trial. J Am Dent Assoc. 2008;139(5):592-7.

25. Paes Leme AF, dos Santos JC, Giannini M, Wada RS. Occlusion of dentin tubules by desensitizing agents. Am J Dent. 2004;17(5):368-72.

26. Ajcharanukul O, Kraivaphan P, Wanachantararak S, Vongsavan N, Matthews B. Effects of potassium ions on dentine sensitivity in man. Arch Oral Biol. 2007;52(7):632-9

27. Yui K, Rodrigues J, Mancini M, Balducci I, Gonçalves S. In vivo evaluation of the effectiveness of bleachingagents on the shade alteration of blood-stained teeth. Int Endod J. 2008;41(6):485-92.

28. Attin T, Paque F, Ajam F, Lennon M. Review of the current status of tooth whitening with the walking bleach technique. Int Endod J. 2003;36(5):313-29.

29. Bispo L, Mondelli J, Clareamento de dentes desvitalizados no consultorio odontologico: uma revisao sobre os aspectos relacionados. $\mathrm{RBO}$ 2005;62(1/2):61-3.

30. Gökay O, Ziramant F, Cali A, Saka $\mathrm{O}$, Radicular peroxide penetration form carbamide peroxide gels during intracoronal bleaching. INT ENDOD J. 2008;41(7):55660

31. Salis, A, Bevilacqua, F, Pozzobon, R. Conclusoes sobre o simposio de clareamento dental. Odontol Clin 1997;7(2):15-6.

32. Lamas C, Angulo G, Alternativa de restauración estética en caso de discromía. Odontol Sanmarquina 2010;13(2):38-41

33. McCaslin A, Haywood V, Potter B, Dickinson G, Russell C. Assessing dentin color changes from nigthguard vital bleaching. J Am Dent Assoc. 1999;130(10):148590.

34. Bernardon J, Sartori N, Ballarin A, Perdigão J, Lopes G, Baratieri L. Clinical performance of vital bleaching techniques. Oper Dent. 2010;35(1):3-10.

35. Dawson P, Sharif M, Smith A, Brunton P. A clinical study comparing the efficacy and sensitivity of home vs combined whitening. Oper Dent. 2011;36(5):460-6. 
36. Ching, H, Palamara, J, Messer H. Effect of hydrogen peroxide and sodium perborate on biomechanical properties of human dentin. J Endod, 2002;28(2):62-7.

37. Portolani J, Candido, M. Efeito dosagentes clareadores sobre as estruturas dentais. Rev Odontol UNESP 2005;34(2):91-4.

38. Soldani P, Amaral CM, Rodrigues JA. Microhardness evaluation of in situ vital bleaching and thickening agents on human dental enamel. Int J Periodontics Restorative Dent. 2010;30:203-11.

39. Eimar H, Siciliano R, Abdallah $\mathrm{MN}$ et al.Hydrogen peroxide whitens teeth by oxidizing the organic structure J Dent. 2012 doi: 10.1016/j.jdent.2012.08.008

40. Attin T, Hannig C, Wiegand A, Attin R. Effect of bleaching on restorative materials and restorations a systematic review. Dent Mat. 2004;20:852-61.

41. Gursoy UK, Eren DI, Bektas OO, Hurmuzlu F, Bostanci V, OzdemirH.Effect of external tooth bleaching on dental plaque accumulation and tooth discoloration.Med Oral Patol Oral Cir Bucal. 2008;13:E266-9

42. Campos I, Briso AL, Pimenta LA, ambrosano G. Effects of bleachingnwithcarbamide peroxide gels on microhardness of restoration materials. J Esthet Restor Dent. 2003;15:175-82.

43. Mello I, Carvalho E, Pollo D, Robazza, C. Avaliacao in vitro da camara pulpar quando da utilizacao do hipoclorito de sodio a $1 \%$ asociado ou nao ao EDTA-T a $17 \%$ e acido fosforico a $37 \%$ para realizacao de clareamento endogeno. J Bras.Endo/Perio. 2000;1(3):7-10.

44. Teixeira E, Teixeira F, Ferraz C. Clareamento dental interno com pasta de perborato de sodio e agua destilada. Rev Assoc. Paul. Cir Dent. 2000;54(4):315-8.

45. Tredwin CJ, Naik S, Lewis NJ, Scully C. Hydrogen peroxide toothwhitening (bleaching) products: review of adverse effects and safety issues. British Dental Journal. 2006;200(7):371-6.
46. Sulieman M.A.M. An overview of tooth-bleaching techniques: chemistry, safety and efficacy. Periodontology 2000, 2008; 48:148-69.

47. Tay LY, Kose C, Loguercio AD, Reis A. Assessing the effect of a desensitizing agent used before inoffice tooth bleaching. J Am Dent Assoc. 2009;140(10):1245-51.

48. Lewinstein I, Fuhrer N, Churaru N, Cardash H. Effect of different peroxide bleaching regimens and subsequent fluoridation on the hardness of human enamel and dentin. J Prosthet Dent. 2004; 92(4):337-42.

49. Brannstrom $M$. The hydrodynamic theory of dentinal pain: sensation in preparations, caries, and the dentinal crack syndrome. J Endod. 1986;12(10):453-7.

50. Sun G. The role of laser in cosmetic dentistry. Dent Clin North Am 2000;44(4):831-50

51. Mondelli RFL. Clareamento de dentes polpados: técnicas e equipamentos. Biodonto 2003;1(1):10-71 